\title{
Order of superconductive phase transition
}

\author{
H.Kleinert \\ Institut für Theoretische Physik, \\ Freie Universität Berlin, \\ Arnimallee 14, D-14195 Berlin, Germany
}

Received December 26, 2004

\begin{abstract}
On the occasion of Reinhard Folk's 60th birthday, I give a brief review of the theoretical progress in understanding the critical properties of superconductors. I point out the theoretical difficulties in finding a second-order transition in the Ginzburg-Landau Model with $\mathrm{O}(\boldsymbol{N})$-symmetry in $4-\varepsilon$ Dimensions, and the success in predicting the existence and location of a tricritical point with the help of a dual disorder theory.
\end{abstract}

Key words: superconductivity, phase transitions, renormalization group

PACS: 05.70.Jk, 64.60.Fr, 74.20.-Z

\section{Introduction}

Among the many important contributions made by Reinhard Folk in the field of critical phenomena is his work on the properties of the superconducting phase transitions [1,2], where he discussed the effect of two-loop contributions on the renormalization flow. To point out its significance, let me briefly recall the historic background of the problem studied by him with various collaborators, most prominently with Yurij Holovatch.

Until thirty years ago, the superconductive phase transition was always assumed to be of second order. All critical exponents had the mean-field values required by the Ginzburg-Landau model. In 1974, however, it was noted by Halperin, Lubensky, and Ma [3] (see also [4]) that the renormalization group treatment of the GinzburgLandau model of superconductivity did not produce a critical point. A similar observation had been published earlier in four-dimensional quantum field theory by Coleman and Weinberg [5] (working at the same institute). This was interpreted as an indication that thermal fluctuations which, in general, cause only small deviations of the critical exponents from their mean-field values, would here have the more dramatic effect of driving the superconductive transition first order. At that time, the theoretical result could not be tested by experiment. The reason is that in 
old-fashioned superconductors, the temperature interval around the critical point, in which fluctuations become important, the so-called Ginzburg interval [6], is extremely narrow, lying in the nK-regime. This explained the observed mean-field behavior. The critical regime had always been too small to be resolved. See the textbooks $[7,8]$ for details.

To probe the Ginzburg interval and confirm the second-order nature of the transition, one had to rely on Monte-Carlo simulations [9] and a theoretical analogy with smectic-nematic transitions in liquid crystals [10], which is also described by a Ginzburg-Landau model, albeit with a different interpretation of the fields [11] (the so-called Landau-De Gennes model).

The situation has changed since the discovery of high- $T_{\mathrm{c}}$ superconductors, where the Ginzburg interval is large, up to several K. Recent experiments [12] clearly show a critical behavior associated with a charged critical point, in which both the coherence length $\xi$ and the magnetic penetration depth $\lambda$ grow with the same critical exponent $\nu_{\lambda}=\nu_{\xi} \approx 2 / 3[13]$.

The confusing critical properties of the Ginzburg-Landau model certainly required further investigation, in particular it appeared necessary to calculate higher loop approximations. To appreciate the problem, let us first recall what makes the first-order result of [3] questionable: The simplest argument leading to the first order is based on a fluctuation-corrected mean-field theory obtained in three dimensions by integrating out exactly the gauge field at fixed $|\phi|$. The resulting trace-log can be evaluated exactly, and produces a cubic term $\propto-|\phi|^{3}$, which makes the transition first-order. The trouble with this argument is that in the type II regime, fluctuations will produce vortex lines. At the core of each vortex line, the order field $\phi$ vanishes, which invalidates the assumption of a fixed $|\phi|$ in the above calculation.

The first direct theoretical evidence for a second-order phase transition did not come from the Ginzburg-Landau model but from a dual formulation of it on a lattice which I set up about 20 years ago [14]. Its properties are discussed in great detail in the textbook [7]. The dual formulation contains no massless gauge field, and this avoids the above problem coming from the zeroth in the order field $\phi$. My dual model predicted definitely a second-order transition in the type-II regime characterized by a Ginzburg parameter $\kappa>1 / \sqrt{2}$. This parameter measures the ratio between magnetic penetration depth and coherence length in the superconductor.

The dual theory contains a different field $\psi$ which is a disorder field. The associated Feynman diagrams are direct pictures of the vortex loops, whose density is $|\psi|^{2}$. This field has a quartic self-interaction accounting for their short-range repulsion, and a gauge-coupling to a massive vector field $\vec{h}$ representing the fluctuating magnetic induction in the superconducting phase. Due to the mass term, the field $\vec{h}$ can be integrated out and the assumption of a constant mean field $|\psi|$ presents no problem, leading to a a Landau-like expansion of the free energy containing terms $|\psi|^{4},|\psi|^{6}$, etc. The $|\psi|^{4}$ term turned out to be proportional to $\kappa-\kappa_{\mathrm{t}}$, with [14]

$$
\kappa_{\mathrm{t}} \approx \frac{3 \sqrt{3}}{2 \pi} \sqrt{1-\frac{4}{9}\left(\frac{\pi}{3}\right)^{4}} \equiv \frac{0.798}{\sqrt{2}}
$$


For $\kappa<\kappa_{\mathrm{t}}$, vortices attract each other on the average, and the transition is of first order, whereas for $\kappa>\kappa_{\mathrm{t}}$, they repel each other and the transition is of second order. Thus, the mean-field approximation in the disorder theory suggests a second-order transition in the type II regime of repulsive vortex lines and a first-order transition in the type I regime of attractive vortex lines.

The point $\kappa=\kappa_{\mathrm{t}}$ is a tricritical point. Its existence has been confirmed by Monte Carlo simulations on a lattice in [15], but initially at quite a different value $\kappa_{\mathrm{t}} \approx$ $0.38 / \sqrt{2}$. Only recently, with the availability of much better simulation techniques, has my 1982 prediction been confirmed with amazing accuracy [16].

In order to satisfactoryly understand the transition, one should be able to explain the second order of the transition in the type-II regime without the duality argument. Thus we must find the effective potential of the Ginzburg-Landau model as accurately as possible. So far, only the two-loop effective potential is known for $N / 2$ complex fields in $4-\varepsilon$ dimensions, which I calculated together with my collaborator Van den Bossche [17]. The result is given in sections 3 and 4. The case $N=2$ concerns the physical situation of the Ginzburg-Landau model of superconductivity.

The involved calculations became possible due to recent progress made in evaluation techniques of Feynman diagrams in $4-\varepsilon$ dimensions. In particular, two-loop Feynman diagrams with unequal masses of the internal lines have become available analytically $[18,19]$. Moreover, the full $\varepsilon$-expansion of the so-called sunset diagram is now known [20]. This is important for critical phenomena in $4-\varepsilon$ dimensions since, for instance, a three-loop calculation requires the knowledge of all terms of order $\varepsilon$ of the two-loop diagrams.

Unfortunately, the renormalization flow of the coupling constants in $4-\epsilon$ dimensions does not improve much over the one-loop calculation of [3] when going to two loops [21,22]. Apparently, a direct three-dimensional approach is more promising to lead to a second-order transition for $N=2$ [23,24]. The absence of a charged fixed point for $N=2$ in $4-\varepsilon$ dimensions seems thus to be a specific weakness of the $\varepsilon$-expansion, although important progress has been made in [1]: There, a [1/1] PadéBorel resummation of the two beta functions (associated with the electric charge and with the self-coupling of the scalar field) has indeed lead to a desired IR stable fixed point for $N=2$. The resummation at such a low order is, however, not very reliable and the result should be considered as fortuitous.

After all this work it is not even certain that a higher-loop renormalization group analysis can really lead to a critical point. In my opinion, the perturbation expansion to low orders knows too little about the vortex fluctuations, which should be included explicitly into the path integral [25], and which the dual theory takes optimally into account. In $\phi^{4}$-theory without gauge field, this seems to be unnecessary for reasons which are not yet understood. The omission is only justified there by the success in predicting the experimental critical exponents in these systems [8].

We hope that eventually variational perturbation theory will be capable of locating a fixed point of the Ginzburg-Landau model, thus allowing us to extract physical values independently of [1]. This theory has been developed and discussed in detail in $[8,26-30]$ and it has proven to be a powerful tool for determining critical exponents 
in three [28] as well as in $4-\varepsilon$ dimensions [30] of pure $\phi^{4}$-theories. Variational perturbation theory is a procedure which allows us to determine resummed quantities from a strong-coupling limit of divergent expansions in powers of the bare coupling constant. It no more requires Padé or Padé-Borel resummation. In particular, there is no freedom in selecting different Padé approximants which yield different results at higher orders than $[1 / 1]$. Variational perturbation theory has yielded the most precise prediction $[26] \alpha \approx-0.0129 \pm 0.0006$ for the best-measured critical exponent $\alpha$ governing the singularity of the specific heat of superfluid helium. To avoid the smear-out of the critical regime by gravity over $10^{-6} \mathrm{~K}$ and to determine the critical temperature up to $2 \mathrm{nK}$, the experiment has been performed in the satellite [31] and gave $\alpha=-0.0127 \pm 0.0003$.

Recently, we have applied variational perturbation theory to a new range of problems: the determination of amplitude ratios in three dimensions of the $\mathrm{O}(N)$ model [32]. We based these calculations on expansions of the critical exponents obtained by a certain regularization method [33], in which analytic regularization is applied with a formal minimal subtraction in $4-\varepsilon$ dimensions, but inserting at the end $\varepsilon=1$ without invoking further $\varepsilon$-expansions. Our results were consistent with Padé-Borel methods. Due to its power, we believe that variational perturbation theory will eventually succeed in producing the desired zeros in the beta functions to yield an IR-stable charged fixed point.

When calculating the effective potential to be given in sections 3 and 4, it was useful to confirm the agreement of our renormalization constants in [17] with those obtained by Reinhard Folk and his collaborator Kolnberger of 1992 [21]. A student of mine had done this calculation eight years earlier for his M.S. thesis [22], but one of his coefficients contained an error, which was corrected in Folk's work.

Let us also mention that the effective potential given in sections 3 and 4 may be seen as an extension to a two-coupling-constants problem studied by Brézin et al. [34] (see also [35]), who give the $\varepsilon$-expansion of the equation of state of the $N$-components $\phi^{4}$ theory without a gauge field up to two loops [36].

\section{Ginzburg-Landau model}

The Lagrangian density contains $N / 2$ complex scalar fields $\phi$ coupled to the abelian fields $A_{\mu}$ and reads, with a covariant gauge fixing,

$$
\mathcal{L}=|D \phi|^{2}+m^{2} \phi^{2}+\frac{g}{3 !}|\phi|^{4}+\frac{1}{4} F_{\mu \nu}^{2}+\frac{1}{2 \alpha}\left(\partial_{\mu} A_{\mu}\right)^{2},
$$

where $D_{\mu}=\partial_{\mu}-\mathrm{i} e A_{\mu}$ is the covariant derivative, $F_{\mu \nu}$ is the usual field-strength tensor, and $\alpha$ is a gauge parameter. In principle, there are also ghost fields which, however, decouple in the symmetric phase and remain massless. Working in dimensional regularization they do not contribute to the energy due to Veltman's rule $\int \mathrm{d}^{D} p p^{\alpha}=0$ for all $\alpha[8]$. The effective potential will be obtained using the so-called background-field method of DeWitt [38]. We shift the scalar field by an unknown constant $\Phi: \phi \rightarrow \Phi+\phi$. This generates new vertices. To simplify the calculation, 
we shall use throughout the Landau gauge $\alpha \rightarrow 0$. This reduces the number of Feynman diagrams and, since $\alpha=0$ enforces $\partial_{\mu} A_{\mu} \equiv 0$ at the Lagrangian level, removes a possible mixing of $A_{\mu} \phi$ and $A_{\mu} \phi^{\dagger}$ terms, thus decoupling scalar and gauge propagators. It is further advantageous to use real fields, defining

$$
\phi=\frac{1}{\sqrt{2}}\left(\phi_{1}+\mathrm{i} \phi_{2}\right), \quad \Phi=\frac{1}{\sqrt{2}}\left(\Phi_{1}+\mathrm{i} \Phi_{2}\right) .
$$

Then the Lagrangian has the expansion around the background field:

$$
\begin{aligned}
\mathcal{L}= & \mathcal{L}_{0}+\frac{1}{2} \phi\left[G^{\mathrm{T}} P^{\mathrm{T}}+G^{\mathrm{L}} P^{\mathrm{L}}\right] \phi+\frac{1}{2} A_{\mu} D^{\mathrm{T}} P_{\mu \nu}^{T} A_{\nu}+\frac{g}{4 !}\left[\left(\phi^{2}\right)^{2}+4 \Phi \phi\left(\phi^{2}\right)\right] \\
& +e^{2} A^{2} \Phi \phi+\frac{1}{2} e^{2} A_{\mu}^{2} \phi^{2}+e A_{\mu}\left(\phi_{2} \partial_{\mu} \phi_{1}-\phi_{1} \partial_{\mu} \phi_{2}\right),
\end{aligned}
$$

where $\phi$ and $\Phi$ are now $N$ components real fields written as two-dimensional isovectors $\phi=\left(\phi_{1}, \phi_{2}\right)$ and $\Phi=\left(\Phi_{1}, \Phi_{2}\right)$. The notation is:

$$
\begin{aligned}
\mathcal{L}_{0} & =\frac{1}{2} m^{2} \Phi^{2}+\frac{g}{4 !} \Phi^{4}, \\
G^{\mathrm{T}} & \equiv-\partial^{2}+m_{\mathrm{T}}^{2}=-\partial^{2}+m^{2}+\frac{g}{3 !} \Phi^{2}, \quad G^{\mathrm{L}} \equiv-\partial^{2}+m_{\mathrm{L}}^{2}=-\partial^{2}+m^{2}+\frac{g}{2} \Phi^{2}, \\
D^{\mathrm{T}} & \equiv-\partial^{2}+m_{\gamma}^{2}=-\partial^{2}+e^{2} \Phi^{2}, \\
P_{i j}^{\mathrm{T}} & =\delta_{i j}-\frac{\Phi_{i} \Phi_{j}}{\Phi^{2}}, \quad P_{i j}^{\mathrm{L}}=\frac{\Phi_{i} \Phi_{j}}{\Phi^{2}}, \quad P_{\mu \nu}^{T}=\delta_{\mu \nu}-\frac{\partial_{\mu} \partial_{\nu}}{\partial^{2}}
\end{aligned}
$$

with $G^{\mathrm{T}}, G^{\mathrm{L}}$ being the transverse and longitudinal inverse propagators of the scalar field, and $D_{\mu \nu}^{\mathrm{T}}$ is the inverse transverse propagator of the photon field. The transversality of the latter is due to the Landau gauge. Note that there is no term $e A_{\mu} \partial_{\mu}\left(\Phi_{2} \phi_{1}-\right.$ $\left.\Phi_{1} \phi_{2}\right)$ which would mix vector and scalar propagators.

So far, all quantities (fields, coupling constants, and masses) are bare quantities. Up to the second order in the loop expansion, divergences show up as poles in $\varepsilon$ up to the order $1 / \varepsilon^{2}$. They have to be removed to have a finite limit $\varepsilon \rightarrow 0$. This is achieved by the renormalization constants

$$
\begin{aligned}
& \phi=\phi_{r} \sqrt{Z_{\phi}}, \quad A_{\mu}=A_{\mu_{r}} \sqrt{Z_{A}}, \\
& m^{2}=m_{r}^{2} \frac{Z_{m^{2}}}{Z_{\phi}}, \quad g=g_{r} \mu^{\varepsilon} \frac{Z_{g}}{Z_{\phi}^{2}}, \quad e=e_{r} \mu^{\varepsilon / 2} \frac{Z_{e}}{Z_{\phi} \sqrt{Z_{A}}}=\frac{e_{r}}{\sqrt{Z_{A}}} \mu^{\varepsilon / 2},
\end{aligned}
$$

where in the last equation, the relation $Z_{e}=Z_{\phi}$ has been used, which is a consequence of a Ward identity. Intuitively, it comes from the requirement $D_{\mu} \phi \rightarrow$ $\sqrt{Z_{\phi}} D_{\mu} \phi_{r}$ for the covariant derivative. In the above equations, the bare quantities are on the left-hand-side and the renormalized ones are on the right-hand-side, indicated by the subscript "r". Note that the vacuum energy requires a special treatment [37].

We now state the result for the effective potential derived in [17] up to two loops. It has the general form

$$
V=V^{(0)}+\frac{\hbar}{(4 \pi)^{2}}\left[V^{(1,0)}+\varepsilon V^{(1, \varepsilon)}\right]+\left[\frac{\hbar}{(4 \pi)^{2}}\right]^{2} V^{(2)} .
$$

The expansion terms will be given in the following section. 


\section{Renormalized effective potential to zero- and one-loop order}

The zero-loop effective potential is trivial:

$$
V(l=0)=\frac{1}{2} m^{2} \Phi^{2}+\frac{g}{4 !} \mu^{\varepsilon} \Phi^{4}+\frac{m^{4}}{g \mu^{\varepsilon}} .
$$

The renormalized one-loop potential is simply a combination of the previous bare zero-order and the trace-log terms:

$$
\begin{aligned}
V(l=1) & =\frac{1}{2} m^{2} \Phi^{2} Z_{m^{2}}^{(1)}+\frac{g}{4 !} \mu^{\varepsilon} \Phi^{4} Z_{g}^{(1)}+\frac{m^{4}}{g \mu^{\varepsilon}} Z_{v}^{(1)}+\frac{\Gamma(1-D / 2)}{(4 \pi)^{D / 2}} \frac{1}{D} \mu^{-\varepsilon} \\
& \times\left[(N-1)\left(m_{\mathrm{T}}^{2}\right)^{2}\left(\frac{\mu^{2}}{m_{\mathrm{T}}^{2}}\right)^{\varepsilon / 2}+\left(m_{\mathrm{L}}^{2}\right)^{2}\left(\frac{\mu^{2}}{m_{\mathrm{L}}^{2}}\right)^{\varepsilon / 2}+(D-1)\left(m_{\gamma}^{2}\right)^{2}\left(\frac{\mu^{2}}{m_{\gamma}^{2}}\right)^{\varepsilon / 2}\right] .
\end{aligned}
$$

The constants $Z_{j}^{(1)}$ are chosen to remove the $\varepsilon$-poles at $D=4$ in the Euler $\Gamma(1-D / 2)$ function. They are

$$
\begin{aligned}
Z_{m^{2}}^{(1)} & =g \frac{(N+2)}{3 \varepsilon} \\
g Z_{g}^{(1)} & =\frac{g^{2}(N+8)+108 e^{4}}{3 \varepsilon} \\
Z_{v}^{(1)} & =g \frac{N}{2 \varepsilon}
\end{aligned}
$$

From this we obtain in the Landau gauge

$$
\begin{aligned}
V^{(0)}= & \frac{1}{2} m^{2} \Phi^{2}+\frac{g}{4 !} \mu^{\varepsilon} \Phi^{4}+\frac{m^{4}}{g \mu^{\varepsilon}}, \\
V^{(1,0)}= & \frac{\mu^{-\varepsilon}}{8}\left\{(N-1) m_{\mathrm{T}}^{4}\left[-3+2 \overline{\ln }\left(m_{\mathrm{T}}^{2}\right)\right]+m_{\mathrm{L}}^{4}\left[-3+2 \overline{\ln }\left(m_{\mathrm{L}}^{2}\right)\right]\right. \\
& \left.+m_{\gamma}^{4}\left[-5+6 \overline{\ln }\left(m_{\gamma}^{2}\right)\right]\right\}, \\
V^{(1, \varepsilon)}= & -\frac{\mu^{-\varepsilon}}{96}\left((N-1) m_{\mathrm{T}}^{4}\left\{21-18 \overline{\ln }\left(m_{\mathrm{T}}^{2}\right)+6\left[\overline{\ln }\left(m_{\mathrm{T}}^{2}\right)\right]^{2}+\pi^{2}\right\}\right. \\
& +m_{\mathrm{L}}^{4}\left\{21-18 \overline{\ln }\left(m_{\mathrm{L}}^{2}\right)+6\left[\overline{\ln }\left(m_{\mathrm{L}}^{2}\right)\right]^{2}+\pi^{2}\right\} \\
& \left.+3 m_{\gamma}^{4}\left\{9-10 \overline{\ln }\left(m_{\mathrm{T}}^{2}\right)+6\left[\overline{\ln }\left(m_{\mathrm{T}}^{2}\right)\right]^{2}+\pi^{2}\right\}\right) .
\end{aligned}
$$

A factor $(4 \pi)^{2}$ is absorbed in the definition of $\hbar$ in (10). The function $\overline{\ln }\left(m^{2}\right)$ is defined by

$$
\overline{\ln }\left(m^{2}\right) \equiv \ln \left(\frac{m^{2}}{\mu^{2}}\right)+\gamma-\ln (4 \pi)
$$




\section{Two-loop contribution}

The cancellation of the poles at the two-loop order gives renormalization coefficients $Z_{m^{2}}^{(2)}, Z_{v}^{(2)}$ which depend on $\overline{\ln }\left(m_{\mathrm{L}}\right), \overline{\ln }\left(m_{\mathrm{T}}\right)$ and $Z_{\phi}^{(1)}$ and a renormalization coefficient $Z_{g}^{(2)}$ which depends on $\overline{\ln }\left(m_{\mathrm{L}}\right), \overline{\ln }\left(m_{\mathrm{T}}\right), \overline{\ln }\left(m_{\gamma}\right)$ and $Z_{\phi}^{(1)}, Z_{A}^{(1)}$. The results are

$$
\begin{aligned}
Z_{m^{2}}^{(2)}= & \frac{(N+2)}{9 \varepsilon^{2}}\left[g^{2}(N+5)-18 g e^{2}+54 e^{4}\right] \\
& -\frac{1}{6 \varepsilon}\left[g^{2}(N+2)-8 g e^{2}(N+2)-6 e^{4}(5 N+1)\right] \\
g Z_{g}^{(2)}= & \frac{1}{9 \varepsilon^{2}}\left[g^{3}(N+8)^{2}-18 g^{2} e^{2}(N+8)+108 g e^{4}(N+8)+108 e^{6}(N+18)\right] \\
& -\frac{1}{9 \varepsilon}\left[g^{3}(5 N+22)-12 g^{2} e^{2}(N+5)-18 g e^{4}(5 N+13)+18 e^{6}(7 N+90)\right], \\
Z_{v}^{(2)}= & g \frac{N}{6 \varepsilon^{2}}\left[-18 e^{2}+g(N+2)\right]+g e^{2} N \frac{2}{\varepsilon} \\
Z_{\phi}^{(1)}= & e^{2} \frac{6}{\varepsilon} \\
Z_{A}^{(1)}= & -e^{2} \frac{N}{3 \varepsilon}
\end{aligned}
$$

where a factor $1 /(4 \pi)^{2}$ is again absorbed in the definition of $\hbar$. Then the renormalized two-loop potential in (10) is

$$
V^{(2)}=\mu^{-\varepsilon} \sum_{i=0}^{2} \sum_{j=0}^{2} \sum_{k=0}^{2} V_{i, j, k}^{(2)}\left[\overline{\ln }\left(m_{\mathrm{T}}^{2}\right)\right]^{i}\left[\overline{\ln }\left(m_{\mathrm{L}}^{2}\right)\right]^{j}\left[\overline{\ln }\left(m_{\gamma}^{2}\right)\right]^{k},
$$

where

$$
\begin{aligned}
V_{0,0,0}^{(2)}= & \frac{1}{432 g m_{\gamma}^{4}}\left[1 8 m _ { \gamma } ^ { 2 } \left(g^{2} m_{\gamma}^{2}\left[(N-1)(N+5) m_{\mathrm{T}}^{4}+18 m_{\mathrm{L}}^{2} m_{\mathrm{T}}^{2}-(2 N+13) m_{\mathrm{L}}^{4}\right]\right.\right. \\
& +g e^{2}\left\{42\left(m_{\mathrm{L}}^{2}-m_{\mathrm{T}}^{2}\right)^{2}\left(m_{\mathrm{L}}^{2}+m_{\mathrm{T}}^{2}\right)-6 m_{\gamma}^{2}\left[(19 N-31) m_{\mathrm{T}}^{4}+24 m_{\mathrm{T}}^{2} m_{\mathrm{L}}^{2}+7 m_{\mathrm{L}}^{4}\right]\right. \\
& \left.\left.-18 m_{\gamma}^{4}\left[(N-1) m_{\mathrm{T}}^{2}+m_{\mathrm{L}}^{2}\right]+(19 N-36) m_{\gamma}^{6}\right\}-108 e^{4} m_{\gamma}^{2}\left(m_{\mathrm{L}}^{2}-m_{\mathrm{T}}^{2}\right)\left[(N-1) m_{\mathrm{T}}^{2}+3 m_{\mathrm{L}}^{2}\right]\right) \\
+ & 6 g \Phi^{2}\left\{7 g^{2} m_{\gamma}^{4}\left[2(N-1) m_{\mathrm{T}}^{2}+(N+8) m_{\mathrm{L}}^{2}\right]+18 e^{4}\left(7 m_{\mathrm{L}}^{6}-7 m_{\mathrm{L}}^{4} m_{\gamma}^{2}+11 m_{\mathrm{L}}^{2} m_{\gamma}^{4}+54 m_{\gamma}^{6}\right)\right\} \\
+ & \pi^{2}\left(g^{2} m_{\gamma}^{4}\left[2(N-1) m_{\mathrm{T}}^{2}+(N+8) m_{\mathrm{L}}^{2}\right]\left[3\left(m_{\mathrm{T}}^{2}-m_{\mathrm{L}}^{2}\right)+g \Phi^{2}\right]\right. \\
& +18 g e^{2} m_{\gamma}^{2}\left\{\left(m_{\mathrm{L}}^{2}-m_{\mathrm{T}}^{2}\right)^{2}\left(m_{\mathrm{L}}^{2}+m_{\mathrm{T}}^{2}\right)+3 m_{\gamma}^{4}\left[(N-1) m_{\mathrm{T}}^{2}+m_{\mathrm{L}}^{2}\right]-9 m_{\gamma}^{6}\right\} \\
& \left.+18 e^{4}\left\{-9 m_{\gamma}^{4}\left(m_{\mathrm{L}}^{2}-m_{\mathrm{T}}^{2}\right)\left[(N-1) m_{\mathrm{T}}^{2}+3 m_{\mathrm{L}}^{2}\right]+g \Phi^{2}\left(2 m_{\mathrm{L}}^{6}-m_{\mathrm{L}}^{4} m_{\gamma}^{2}+5 m_{\mathrm{L}}^{2} m_{\gamma}^{4}+10 m_{\gamma}^{6}\right)\right\}\right) \\
+ & 216(4 \pi)^{4} g e^{2}\left[m_{\gamma}^{2}\left(m_{\mathrm{L}}^{2}-m_{\mathrm{T}}^{2}\right)^{2} I^{(0)}\left(m_{\mathrm{L}}, m_{\mathrm{T}}, 0\right)+e^{2} \Phi^{2}\left(m_{\gamma}^{2}-m_{\mathrm{L}}^{2}\right)^{2} I^{(0)}\left(m_{\gamma}, m_{\mathrm{L}}, 0\right)\right] \\
+ & 54 g e^{2}\left(m _ { \gamma } ^ { 2 } \left\{(N-2)\left(m_{\gamma}^{2}-4 m_{\mathrm{T}}^{2}\right) m_{\gamma}^{2} \xi\left(m_{\gamma}, m_{\mathrm{T}}, m_{\mathrm{T}}\right)\right.\right. \\
& \left.+2\left[m_{\mathrm{T}}^{4}-2 m_{\mathrm{T}}^{2}\left(m_{\gamma}^{2}+m_{\mathrm{L}}^{2}\right)+\left(m_{\gamma}^{2}-m_{\mathrm{L}}^{2}\right)^{2}\right] \xi\left(m_{\gamma}, m_{\mathrm{T}}, m_{\mathrm{L}}\right)\right\}
\end{aligned}
$$




$$
\begin{aligned}
& \left.+e^{2} \Phi^{2}\left(m_{\mathrm{L}}^{4}-4 m_{\mathrm{L}}^{2} m_{\gamma}^{2}+12 m_{\gamma}^{4}\right) \xi\left(m_{\gamma}, m_{\gamma}, m_{\mathrm{L}}\right)\right) \\
& \left.+6 g^{3} \Phi^{2} m_{\gamma}^{4}\left[(N-1) \xi\left(m_{\mathrm{T}}, m_{\mathrm{T}}, m_{\mathrm{L}}\right)+3 \xi\left(m_{\mathrm{L}}, m_{\mathrm{L}}, m_{\mathrm{L}}\right)\right]\right], \\
& V_{1,0,0}^{(2)}=-\frac{m_{\mathrm{T}}^{2}}{12 g m_{\gamma}^{2}}\left(g^{2}(N-1) m_{\gamma}^{2}\left[-m_{\mathrm{L}}^{2}+(N+3) m_{\mathrm{T}}^{2}+2 g \Phi^{2}\right]\right. \\
& \left.+6 g e^{2}\left\{3\left(m_{\mathrm{L}}^{2}-m_{\mathrm{T}}^{2}\right)^{2}-m_{\gamma}^{2}\left[2 m_{\mathrm{L}}^{2}+m_{\mathrm{T}}^{2}(7 N-9)\right]+5(N-1) m_{\gamma}^{4}\right\}-54 e^{4}(N-1) m_{\gamma}^{2}\left(m_{\mathrm{L}}^{2}-m_{\mathrm{T}}^{2}\right)\right), \\
& V_{2,0,0}^{(2)}=\frac{1}{72 g m_{\gamma}^{2}}\left(g^{2}(N-1) m_{\gamma}^{2}\left\{-m_{\mathrm{L}}^{2}\left(6 m_{\mathrm{T}}^{2}+g \Phi^{2}\right)+m_{\mathrm{T}}^{2}\left[3 m_{\mathrm{T}}^{2}(N+3)+4 g \Phi^{2}\right]\right\}\right. \\
& -9 g e^{2}\left[-2\left(m_{\mathrm{L}}^{2}-m_{\mathrm{T}}^{2}\right)^{2} m_{\mathrm{T}}^{2}+m_{\gamma}^{2}(N-2)\left(m_{\gamma}^{4}+6 m_{\mathrm{T}}^{4}\right)-6(2 N-3) m_{\gamma}^{4} m_{\mathrm{T}}^{2}\right] \\
& \left.-162 e^{4}(N-1) m_{\gamma}^{2}\left(m_{\mathrm{L}}^{2}-m_{\mathrm{T}}^{2}\right) m_{\mathrm{T}}^{2}\right) \text {, } \\
& V_{0,1,0}^{(2)}=-\frac{m_{\mathrm{L}}^{2}}{12 g m_{\gamma}^{4}}\left(g^{2} m_{\gamma}^{4}\left[-(N+5) m_{\mathrm{L}}^{2}+(2 N+7) m_{\mathrm{T}}^{2}+(N+8) g \Phi^{2}\right]\right. \\
& +6 g e^{2} m_{\gamma}^{2}\left\{3\left(m_{\mathrm{L}}^{2}-m_{\mathrm{T}}^{2}\right)^{2}-m_{\gamma}^{2}\left[5 m_{\mathrm{L}}^{2}+2 m_{\mathrm{T}}^{2}\right]+5 m_{\gamma}^{4}\right\} \\
& \left.-6 e^{4}\left\{m_{\gamma}^{4}\left[27\left(m_{\mathrm{L}}^{2}-m_{\mathrm{T}}^{2}\right)-16 g \Phi^{2}\right]+3 g \Phi^{2} m_{\mathrm{L}}^{2}\left(2 m_{\gamma}^{2}-m_{\mathrm{L}}^{2}\right)\right\}\right), \\
& V_{0,2,0}^{(2)}=\frac{m_{\mathrm{L}}^{2}}{72 g m_{\gamma}^{4}}\left(g^{2} m_{\gamma}^{4}\left[-3(N+5) m_{\mathrm{L}}^{2}+3(N+8) m_{\mathrm{T}}^{2}+(N+17) g \Phi^{2}\right]\right. \\
& +18 g e^{2} m_{\gamma}^{2}\left[\left(m_{\mathrm{L}}^{2}-m_{\mathrm{T}}^{2}\right)^{2}+3 m_{\gamma}^{4}\right] \\
& \left.-9 e^{4}\left\{2 m_{\gamma}^{4}\left[27\left(m_{\mathrm{L}}^{2}-m_{\mathrm{T}}^{2}\right)-7 g \Phi^{2}\right]+g \Phi^{2} m_{\mathrm{L}}^{2}\left(4 m_{\gamma}^{2}-3 m_{\mathrm{L}}^{2}\right)\right\}\right), \\
& V_{0,0,1}^{(2)}=\frac{e^{2}}{6 m_{\gamma}^{2}}\left\{-9 e^{2} m_{\mathrm{L}}^{4} \Phi^{2}-3 m_{\gamma}^{2}\left[m_{\mathrm{L}}^{4}+m_{\mathrm{T}}^{4}-2 m_{\mathrm{L}}^{2}\left(m_{\mathrm{T}}^{2}+4 e^{2} \Phi^{2}\right)\right]\right. \\
& \left.+12 m_{\gamma}^{4}\left[m_{\mathrm{L}}^{2}+(N-1) m_{\mathrm{T}}^{2}-6 e^{2} \Phi^{2}\right]-m_{\gamma}^{6}(4 N-9)\right\}, \\
& V_{0,0,2}^{(2)}=\frac{e^{2}}{8 m_{\gamma}^{4}}\left[-18 m_{\gamma}^{8}+e^{2} \Phi^{2}\left(-m_{\mathrm{L}}^{6}+8 m_{\mathrm{L}}^{4} m_{\gamma}^{2}-22 m_{\mathrm{L}}^{2} m_{\gamma}^{4}+40 m_{\gamma}^{6}\right)\right] \text {, } \\
& V_{1,1,0}^{(2)}=\frac{1}{36 m_{\gamma}^{2}}\left\{g(N-1) m_{\mathrm{L}}^{2} m_{\gamma}^{2}\left(3 m_{\mathrm{T}}^{2}+g \Phi^{2}\right)\right. \\
& \left.-9 e^{2}\left[-\left(m_{\mathrm{L}}^{2}-m_{\mathrm{T}}^{2}\right)^{2}\left(m_{\mathrm{L}}^{2}+m_{\mathrm{T}}^{2}\right)+3 m_{\gamma}^{2}\left(m_{\mathrm{L}}^{4}+m_{\mathrm{T}}^{4}\right)-3 m_{\gamma}^{4}\left(m_{\mathrm{L}}^{2}+m_{\mathrm{T}}^{2}\right)+m_{\gamma}^{6}\right]\right\}, \\
& V_{1,0,1}^{(2)}=\frac{e^{2}}{4 m_{\gamma}^{2}}\left[-\left(m_{\mathrm{L}}^{2}-m_{\mathrm{T}}^{2}\right)^{3}+3 m_{\gamma}^{2}\left(m_{\mathrm{L}}^{4}-m_{\mathrm{T}}^{4}\right)-3 m_{\gamma}^{4}\left(m_{\mathrm{L}}^{2}-m_{\mathrm{T}}^{2}\right)+(N-1) m_{\gamma}^{6}\right] \text {, } \\
& V_{0,1,1}^{(2)}=\frac{e^{2}}{4 m_{\gamma}^{4}}\left\{e^{2} m_{\mathrm{L}}^{6} \Phi^{2}+m_{\gamma}^{2}\left[m_{\mathrm{L}}^{6}-m_{\mathrm{L}}^{4}\left(3 m_{\mathrm{T}}^{2}+4 e^{2} \Phi^{2}\right)+3 m_{\mathrm{L}}^{2} m_{\mathrm{T}}^{4}-m_{\mathrm{T}}^{6}\right]\right. \\
& \left.+m_{\gamma}^{4}\left[m_{\mathrm{L}}^{2}\left(-3 m_{\mathrm{L}}^{2}+14 e^{2} \Phi^{2}\right)+3 m_{\mathrm{T}}^{4}\right]+3 m_{\gamma}^{6}\left(m_{\mathrm{L}}^{2}-m_{\mathrm{T}}^{2}\right)+m_{\gamma}^{8}\right\} .
\end{aligned}
$$

The function $I^{(0)}\left(m_{1}, m_{2}, 0\right)$ in $(26)$ denotes a non-diverging piece, without the coefficient $\left(\mu^{\varepsilon}\right)^{2}$, of

$$
\begin{aligned}
\left(\mu^{\varepsilon}\right)^{2}(4 \pi)^{4} I\left(m_{1}, m_{2}, 0\right)=-\frac{2}{\varepsilon^{2}}\left(m_{1}^{2}+m_{2}^{2}\right)-\frac{2}{\varepsilon}\left[\frac{3}{2}\left(m_{1}^{2}+m_{2}^{2}\right)-L_{1}\left(m_{1}, m_{2}, 0\right)\right] \\
-\frac{1}{2}\left\{L_{2}\left(m_{1}, m_{2}, 0\right)-6 L_{1}\left(m_{1}, m_{2}, 0\right)+2 m_{1}^{2} \overline{\ln }\left(m_{1}^{2}\right) \overline{\ln }\left(m_{2}^{2}\right)+\left[\overline{\ln }\left(m_{1}^{2}-m_{2}^{2}\right)\right]^{2}\left(m_{1}^{2}-m_{2}^{2}\right)\right.
\end{aligned}
$$




$$
\begin{aligned}
& -2 \overline{\ln }\left(m_{1}^{2}-m_{2}^{2}\right) \overline{\ln }\left(m_{2}^{2}\right)\left(m_{1}^{2}-m_{2}^{2}\right)+2\left(m_{1}^{2}-m_{2}^{2}\right) \operatorname{Li}_{2}\left(\frac{m_{2}^{2}}{m_{2}^{2}-m_{1}^{2}}\right)+\left(m_{1}^{2}+m_{2}^{2}\right)[7+\zeta(2)] \\
& \left.+\frac{\pi^{2}}{3}\left(m_{1}^{2}-m_{2}^{2}\right)\right\}
\end{aligned}
$$

where

$$
\begin{aligned}
& L_{1}\left(m_{1}, m_{2}, m_{3}\right)=m_{1}^{2} \overline{\ln }\left(m_{1}^{2}\right)+m_{2}^{2} \overline{\ln }\left(m_{2}^{2}\right)+m_{3}^{2} \overline{\ln }\left(m_{3}^{2}\right), \\
& L_{2}\left(m_{1}, m_{2}, m_{3}\right)=m_{1}^{2}\left[\overline{\ln }\left(m_{1}^{2}\right)\right]^{2}+m_{2}^{2}\left[\overline{\ln }\left(m_{2}^{2}\right)\right]^{2}+m_{3}^{2}\left[\overline{\ln }\left(m_{3}^{2}\right)\right]^{2},
\end{aligned}
$$

The function $\xi\left(m_{1}, m_{2}, m_{3}\right)$ in $(26)$ is defined by

$$
\begin{aligned}
\xi\left(m_{1}, m_{2}, m_{3}\right) \equiv & 4\left(2 m_{1}^{2} m_{2}^{2}+2 m_{1}^{2} m_{3}^{2}+2 m_{2}^{2} m_{3}^{2}-m_{1}^{4}-m_{2}^{4}-m_{3}^{4}\right)^{1 / 2} \\
& \times\left[L\left(\theta_{1}\right)+L\left(\theta_{2}\right)+L\left(\theta_{3}\right)-\frac{\pi}{2} \ln (2)\right]
\end{aligned}
$$

where $L(t)$ is the Lobachevsky function, defined by

$$
L(t)=-\int_{0}^{t} \mathrm{~d} x \ln \cos x
$$

and the angles are given by

$$
\theta_{j}=\arctan \left[\frac{\left(m_{1}^{2}+m_{2}^{2}+m_{3}^{2}\right)-2 m_{j}^{2}}{\left(2 m_{1}^{2} m_{2}^{2}+2 m_{1}^{2} m_{3}^{2}+2 m_{2}^{2} m_{3}^{2}-m_{1}^{4}-m_{2}^{4}-m_{3}^{4}\right)^{1 / 2}}\right] .
$$

\section{Conclusion}

As should have become clear from this short review, the superconductive phase transition still poses many interesting problems. In particular, the inclusion of vortex loop fluctuations into the ordinary $\epsilon$-expansion seems necessary to stabilize the renormalization flow in the type-II regime. I hope that after his 60 th birthday, Reinhard Folk will have many more active years to contribute to our understanding of this theoretically and experimentally very important phase transition.

\section{Acknowledgements}

I am grateful to Dr. F.S.Nogueira for many interesting discussions and to the European Network COSLAB for partial support. 


\section{References}

1. Folk R., Holovatch Yu., J. Phys. A, 29, 1996, 3409.

2. Folk R., Holovatch Yu., Critical Fluctuations in Normal-to-Superconducting Transition. Correlations, Coherence, and Order, ed. by Shopova D.V., Uzunov D.I. Kluwer Academic/Plenum Publishers, N.Y.-London, 1999, 83-116; cond-mat/9807421.

3. Halperin B.I., Lubensky T.C., Ma S.-K., Phys. Rev. Lett., 1974, 32, 292;

Chen J.-H., Lubensky T.C., Nelson D.R., Phys. Rev. B, 1978, 17, 4274.

4. Lawrie I.A., Nucl. Phys. B, 1982, 200, 1.

5. Coleman S., Weinberg E., Phys. Rev. D, 1973, 7, 1888;

Kleinert H. Limitations on Coleman-Weinberg mechanism. Phys. Lett. B, 1983, 128, 69, (http://www.physik.fu-berlin.de/ ${ }^{k} k$ leinert/106).

6. Ginzburg V.L., Fiz. Twerd. Tela, 1960, 2, 2031;

Sov. Phys. Solid State, 1961, 2, p. 1824;

see also the detailed discussion in Chapter 13 of the textbook L.D. Landau and E.M. Lifshitz, Statistical Physics, 3rd edition. Pergamon Press, London, 1968.

Note, however, that the Ginzburg criterion is not applicable to systems with continuous symmetry. It specifies where fluctuations drive an order parameter across a barrier separating two phases. In systems with continuous symmetry, the order goes around a barrier. This is much easier, in particular, if the symmetry is high [factor $N^{2}$ for $\mathrm{O}(N)$-symmetry]. The proper criterion is derived in

Kleinert H. Criterion for dominance of directional versus size fluctuations of order field in restoring spontaneously broken continuous symmetries. Phys. Rev. Lett., 2000, 84, 286; (cond-mat/9908239)

7. Kleinert H. Gauge Fields in Condensed Matter, vol. 1, p. 735-742. Superflow and Vortex Lines. World Scientific Publishing Co., Singapore, 1989, (http://www .physik.fu-berlin.de/ kleinert/b1).

8. Kleinert H., Schulte-Frohlinde V. Critical Properties of $\Phi^{4}$-Theories, p. 1-487. World Scientific, Singapore, 2001, (http://www.physik.fu-berlin.de/ kleinert/b8).

9. Dasgupta C., Halperin B.I., Phys. Rev. Lett., 1981, 47, p. 1556.

10. Als-Nielsen J., Litster J.D., Birgeneau R.J., Kaplan M., Safinya C.R., LindegaardAndersen A., Mathiesen S., Phys. Rev. B, 1980, 22, p. 312.

11. de Gennes P.D., Phys. Lett. A, 1969, 30, 454;

Mol. Cryst. Liq. Cryst., 1971, 12, 193;

The Physics of Liquid Crytsals, p. 85. Oxford University Press, Oxford, 1993;

see also the dual theory in this system:

Kleinert H., J. Phys. (Paris), 1983, 44, 353,

(http://www.physik.fu-berlin.de/ kleinert/102)

12. Schneider T., Khasanov R., Conder K., Pomjakushina E., Bruetsch R., Keller H. Evidence for charged critical fluctuations in underdoped Y-123, (cond-mat/0406691); Schneider T., Khasanov R., Keller H. Evidence for charged critical behavior in the pyrochlore superconductor RbOs2O6, (cond-mat/0409398).

13. A theoretical alternative would be the uncharged critical point in which $\nu_{\lambda}=\nu_{\xi} / 2 \approx$ $1 / 3$. See $[23,24]$.

14. Kleinert H., Lett. Nuovo Cimento, 1982, 35, 405, (http://www.physik. fu-berlin.de/ kleinert/97).

15. Bartholomew J., Phys. Rev. B, 1983, 28, 5378; 
Muneshisa Y., Phys. Lett. B, 1985, 155, 159.

16. Mo S., Hove J., Sudbo A., The order of the metal to superconductor transition, Phys. Rev. B, 2002, 65, 104501, (cond-mat/0109260).

17. Kleinert H., Van den Bossche B., Two-Loop Effective Potential of O(N)-Symmetric Scalar QED in 4-epsilon Dimensions, Nucl. Phys. B, 2002, 632, 51, (condmat/0104102)

18. Ford C., Jack I., Jones D.R.T., Nucl. Phys. B, 1992, 387, 373.

19. Davydychev A.I., Tausk J.B., Nucl. Phys. B, 1993, 397, 123.

20. Davydychev A.I., Phys. Rev. D, 2000, 61, 087701.

21. Kolnberger S., Folk R., Phys. Rev. B, 1992, 41, 4083.

22. Tessman J.-P. Two-loop Renormierung der skalaren Elektrodynamik. Diplomarbeit, Freie Universität, Berlin, 1984, written under my. A pdf file of the thesis is available in the internet: http://www.physik.fu-berlin.de/ kleinert/MS-Tessmann.pdf.

23. Kiometzis M., Kleinert H., Schakel A.M.J., Phys. Rev., 1994, 73, 1975;

Herbut I.F., Tesanović Z., Phys. Rev. Lett., 1996, 76, 4588;

de Calan C., Malbuisson A.P.C., Nogueira F.S., Svaiter N.F., Phys. Rev. B, 1999, 59, 554 ;

de Calan C., Nogueira F.S., Phys. Rev. B, 1999, 60, 4255;

Bergerhoff B., Freire F., Litim D.F., Lola S., Wetterich C., Phys. Rev. B, 1996, 53, 5734 .

24. Kleinert H., Nogueira F.S. Charged fixed point in the Ginzburg-Landau superconductor and the role of the Ginzburg parameter $\kappa$, Nucl. Phys. B, 2003, 651, 361 (this paper shows that a charged fixed point is found in $D=3$ dimensions if $\kappa>1 / \sqrt{2}$ ).

25. Only the knowledge of very high orders gives information on such nonperturbative contributions by appropriate resummation. See

Hamprecht B., Kleinert H., Variational Perturbation Theory for Summing Divergent Non-Borel-Summable Tunneling Amplitudes, J. Phys. A: Math. Gen., 2004, 37, 8561, (hep-th/0303163);

Phys. Lett. B, 2003, 564, 111, (hep-th/0302124).

26. Kleinert H., Phys. Rev. D, 1999, 60, 085001.

27. Kleinert H., Path Integrals in Quantum Mechanics, Statistics and Polymer Physics, (variational perturbation theory is developed in chapters 5 and 17). World Scientific.

28. Kleinert H., Phys. Rev. D, 1998, 57, 2264;

Addendum, 1998, 58, 107702.

29. Jasch F., Kleinert H., J. Math. Phys., 2001, 42, 52.

30. Kleinert H., Phys. Lett. B, 1998, 434, 74;

Phys. Lett. B, 1999, 463, 69;

Kleinert H., Schulte-Frohlinde V., J. Phys. A, 2001, 34, 1037.

31. Lipa J.A., Nissen J.A., Stricker D.A., Swanson D.R., Chui T.C.P., Phys. Rev. B, 2003, 68, 174518, (cond-mat/0310163).

32. Kleinert H., Van den Bossche B., Phys. Rev. E, 2001, 63, 056113.

33. Strösser M., Larin L.A., Dohm V., Nucl. Phys. B, 1999, 540, 654.

34. Brézin E., Wallace D.J., Wilson K.G., Phys. Rev. Let., 1972, 29, 591;

Phys. Rev. B, 1973, 7, 232.

35. Brézin E., Le Guillou J.C., Zinn-Justin J. Phase Transition and Critical Phenomana, edited by C.Domb and M.S.Green, vol. 6., p. 125. Academic Press, New-York, 1976.

36. For the Ising model, corresponding to $N=1$, there exists an $\varepsilon$-expansion of the 
equation of state up to three-loop order in

Wallace D.J., Zia R.K.P., Phys. Lett. A, 1973, 46, 261;

J. Phys. C, 1974, 7, 3480.

37. Kastening B., Kleinert H., Van den Bossche B. Three-Loop Ground-State Energy of $\mathrm{O}(\mathrm{N})-$ Symmetric Ginzburg-Landau Theory above $T_{\mathrm{c}}$ in $4-\epsilon$ Dimensions with Minimal Subtraction, Phys. Rev. B, 2002, 65, 174512, (cond-mat/0109372).

38. de Witt B., Phys. Rev., 1967, 162, 1195.

39. Nogueira F.S., Europhys. Lett., 1999, 45, 612;

de Calan C., Nogueira F.S., Phys. Rev. B, 1999, 60, 11929.

40. Kleinert H., Schakel A., in preparation.

\section{Порядок фазового переходу в надпровідний стан}

\section{Г.Кляйнарт}

Інститут теоретичної фізики,

Вільний університет Берліну,

Арнімале 146 D-14195 Берлін, Німеччина

Отримано 26 грудня 2004 р.

3 нагоди 60-річчя Райнгарда Фолька я приводжу короткий опис теоретичного прогресу в розумінні критичних властивостей надпровідників. Я відзначаю теоретичні труднощі у виявленні фазового переходу другого роду в $\mathrm{O}(\boldsymbol{N})$ симетричній моделі Гінзбурга-Ландау в вимірності $4-\varepsilon$, а також успіх в передбаченні його існування і визначенні трикритичної точки за допомогою дуальної невпорядкованої теорії.

Ключові слова: надпровідність, фазові переходи, ренормалізаційна група

PACS: 05.70.Jk, 64.60.Fr, 74.20.-z 